\title{
Torque Model with Fast and Slow Temperature Dynamics of a Slipping Dry Clutch
}

\author{
Andreas Myklebust, Lars Eriksson \\ Vehicular Systems, Dept. of Electrical Engineering, Linköping University, Sweden \\ Email: andmy@isy.liu.se, larer@isy.liu.se
}

\begin{abstract}
The transmitted torque in a slipping dry clutch is studied in experiments with a heavy duty truck. It is shown that the torque characteristic has little or no dependence on slip speed, but that there are two dynamic effects that make the torque vary up to $900 \mathrm{Nm}$ for the same clutch actuator position. Material expansion with temperature can explain both phenomena and a dynamic clutch temperature model with two different time constants is developed. The dynamic model is validated in experiments, with an error of only $3 \%$ of the maximum engine torque, and is shown to improve the behavior significantly compared to a static model.
\end{abstract}

\section{INTRODUCTION}

Increasing demands on comfort, performance, and fuel efficiency in vehicles lead to more complex transmission solutions. Historically high efficiency was best met with a classical Manual Transmission and comfort with a classical Automatic Transmission. The Automated Manual Transmission (AMT) is one way to combine the best from two worlds. An important part in an AMT is clutch control that has a profound effect on vehicle performance. Therefore it is of importance to know the torque transmitted in the clutch with high precision. Models have come to play an important role in estimation and control of the transmitted torque, since torque sensors are expensive.

A sketch of a dry single-plate clutch is found in Fig. 1, while in-depth explanations are found in for example [1] and [2]. In clutch-modeling literature a wide range of models are proposed. The most simple models have a clutch torque that is assumed to be a controllable input, see for example [3], [4]. These models rely on the assumption that there is perfect knowledge of how the clutch behaves. More advanced models include submodels for slipping and sticking torques. For example a LuGre model is used in [5] and a Karnopp model in [6]. The former is a one-state model that captures stick-slip behavior, varying break-away force, Stribeck effect, and viscous friction. The latter simply applies a dead-zone around zero speed to ease the simulation of stick-slip behavior.

Models for the clutch torque during slipping commonly use a function with the following structure,

$$
M_{c}=\operatorname{sgn}(\Delta \omega) \mu R_{e} F_{N}
$$

where $\Delta \omega$ is the clutch slip (speed), $\mu$ the friction coefficient, $R_{e}$ the effective radius and $F_{N}$ the clamping (normal) force. In these models $F_{N}$ is often either given as input or a static nonlinear function of clutch position, $x$, i.e. $F_{N}=F_{N}(x)$, see for example [2], [7]. In particular [8] mentions that a thirdorder polynomial is suitable to describe the connection between throwout-bearing position and clutch transmitted torque, mainly governed by the flat (cushion) spring characteristics. Furthermore a slight speed dependency of the normal force is hinted in [8], centrifugal forces acting on the springs in the clutch. [1] reports of hysteresis in the diaphragm spring, that could lead to hysteresis in the normal force. In [9] a temperature and wear dependency of the normal force/bearing position characteristics is shown.

Concerning the other model components it is generally recognized that $\mu$ can depend on temperature, slip speed, and wear and that $R_{e}$ can depend on temperature and wear as well, see [10]. In [2] a slip speed dependency of $\mu R_{e}$ is shown, this was especially pronounced for slip speeds below $\sim 100$ RPM.

It can be difficult to separate which parameter in (1) that is the reason for a change in $M_{c}$. Therefore the clutch torque is often studied as a lumped model. In [10] $M_{c}$ is seen to decrease with temperature and $\Delta \omega$. However there are large variations with wear. In [11] $M_{c}$ initially decreases with temperature for low temperatures and then increases for medium and high temperatures. Similarly there are variations with wear and in addition temperature-torque hysteresis are reported. In [12] and [13] temperature models are established, but no work seems to include the temperature effect on $M_{c}$. In view of this the main contribution in this paper is the analysis and model of a clutch that includes temperature dynamics and the corresponding effects on the transmitted torque.

The clutch model presented here is built solely from the observation made on the data presented in this paper. No slip dependency is seen and the torque is increasing with temperature. A limitation of the problem statement is that the clutch is only studied when it is slipping.

\section{EXPERIMENTAL SETUP}

The AMT clutch in a Heavy Duty Truck (HDT) is studied. The clutch, that is in focus here, is a dry single-plate pull-type normally closed clutch. A schematic is seen in Fig. 1. The clutch is actuated by an electric motor that through a worm gear moves a hydraulic master cylinder that in turn moves a slave piston and via a lever pulls the throwout bearing. Both motor position, $x_{m}$, and slave position, $x$, are measured.

The truck used here is only equipped with production sensors, engine speed, idle shaft speed and slave position. Hence no exact torque measurement, is available. In order to 


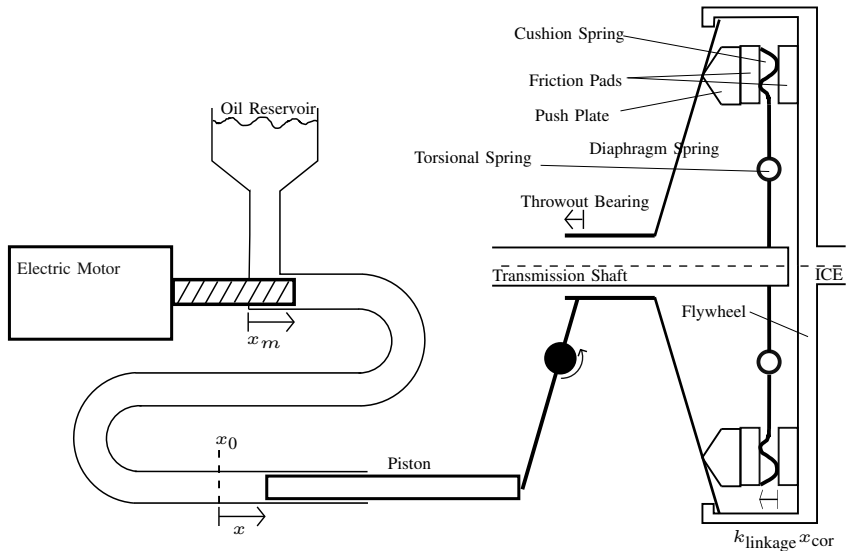

Fig. 1. A schematic over the actuator and the dry single-plate pull-type clutch studied here. $k_{\text {linkage }}$ is the combined ratio of all levers between the piston and the push plate.

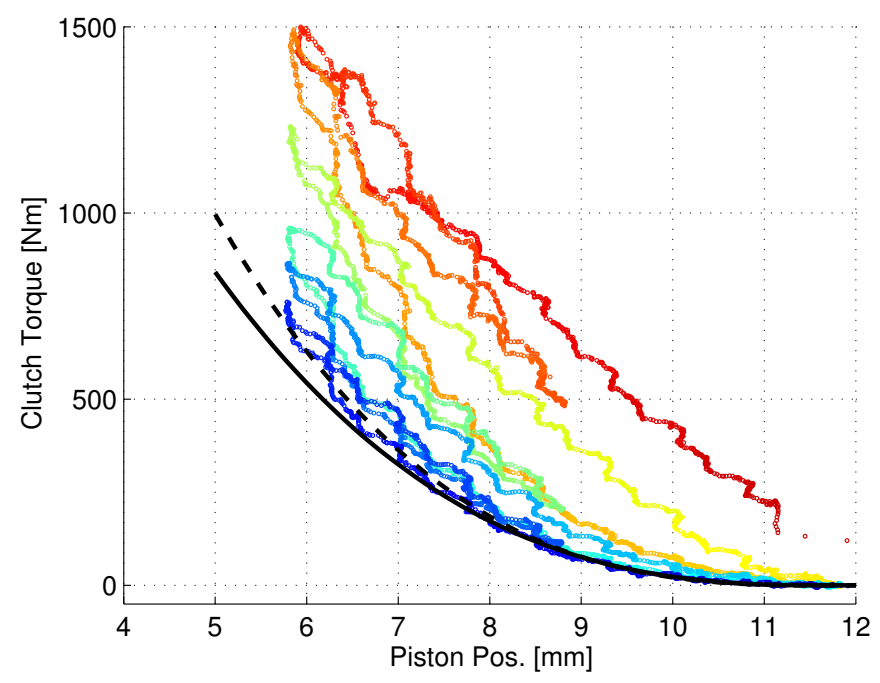

Fig. 2. The clutch position has been ramped back and forth while the clutch torque has been measured. The transmitted torque clearly depends on something more than the clutch position, in particular there is a drift with time (the color changes with time, blue $=0 \mathrm{~s}$ and red $=75 \mathrm{~s}$ ). The torque difference between the first and last ramp is up to $900 \mathrm{Nm}$. The black lines are two parameterizations of (5)

get a measurement of the clutch torque the reported engine torque has been compensated for inertia effects of the engine and flywheel. The limited amount of sensors add to the difficulty in making a clutch model and validating it.

\section{Modeling Outline}

As mentioned in Section I, $M_{c}$ depends on several factors such as, actuator position, temperatures, rotational speeds and wear. In Fig. 2 it is seen how the transmitted torque varies with up to $900 \mathrm{Nm}$ for a given position. Furthermore components are non-linear e.g. spring characteristics, hysteresis, etc. However during experiments no clear hysteresis have been detected and therefore not modeled. Due to the difficulties involved in measuring wear, especially when access to test equipment is limited and non-exclusive, wear has been assumed slow enough to not have a significant effect in the experiments. The only exception is the parameter $x_{0 \text {,ref }}$ of Section $\mathrm{V}$, which is directly affected by the thickness of the friction pads. $x_{0, \text { ref }}$ had to be parameterized to each measurement session (months apart). In an on-line application of the model, such as an observer or controller, more parameters would probably need to be adapted continuously.

In the collected measurement $\Delta \omega>0$ has always hold, hence the sgn-function from (1) has not been considered. And since the clutch is only considered during slipping no advanced friction model, e.g. LuGre, is needed as they tend to be focused around lock up. All these simplifications leave the model structure at

$$
M_{c}=\mu(\Delta \omega, T) R_{e}(T) F_{N}(x, T, \Delta \omega)
$$

In Section $\mathrm{V}$ the temperature effect will be explained by a changing $F_{N}$, at least for medium temperatures of the clutch. Nevertheless with the available sensors there is no way to truly separate the effects between the parameters. Therefore the model structure will be,

$$
M_{c}=M_{c}(x, T, \Delta \omega)
$$

In the next section the slip dependency will be investigated and in the subsequent section the position and temperature dependency will be handled.

\section{SLIP DEPENDENCY}

Slip speed, $\Delta \omega$, dependency is recognized in the literature, [2], [10]. Therefore experiments have been carried out to investigate this. Remember that since this work is limited to when the clutch is slipping, slip speeds have been relatively large. There has been no investigation of what happens when the clutch approaches lock up.

The experiments have been carried out as follows. The truck has been driven to an uphill and has been kept stationary by slipping the clutch at a constant position. This way the vehicle will accelerate forwards if more torque is transmitted and backwards if less torque is transmitted. Furthermore it is easy to initially keep the input shaft speed stationary this way, and by doing so the engine speed can be used to control the slip speed. Slip-speed steps are made and an abrupt change in torque/input shaft acceleration should be seen if there is a slipspeed dependency, Fig. 3. Note that the engine torque will see a transient during the flank of a step due to the acceleration of the engine. A smaller transient is present in the clutch torque signal due to imperfections in the compensation for the acceleration. No significant change is seen in the input shaft acceleration and no correlation between steady-state clutch torque and slip speed is seen neither, Fig 4. Therefore it is assumed that there is no slip dependency at the slip speeds of interest in this paper. Equation (3) can be reduced to

$$
M_{c}=M_{c}(x, T)
$$

This is somewhat in contrast to the data in [10] where a slip dependency is shown. The slip has only been investigated at relatively large speeds and does therefore not contrast the data in [2]. 

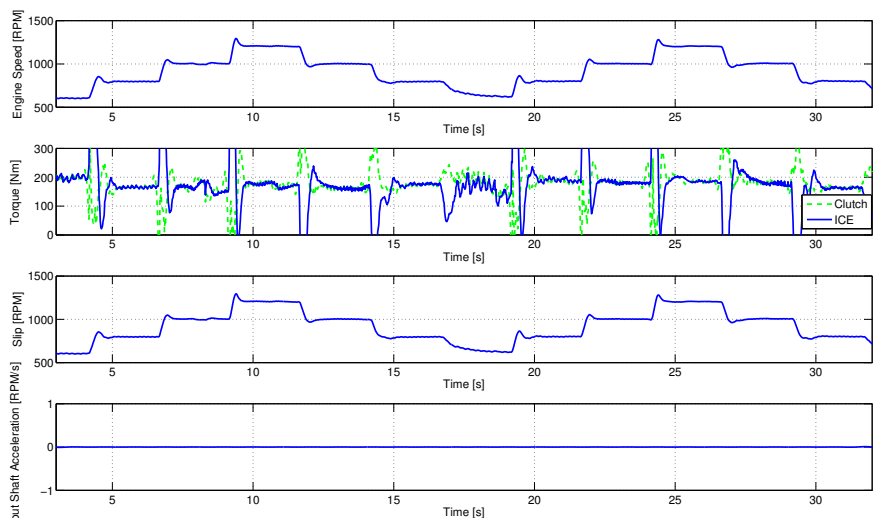

Fig. 3. Slip steps are made through engine-speed control when the truck is held stationary in a slight uphill using a constant clutch position. No reaction is seen in neither clutch torque or input shaft acceleration.

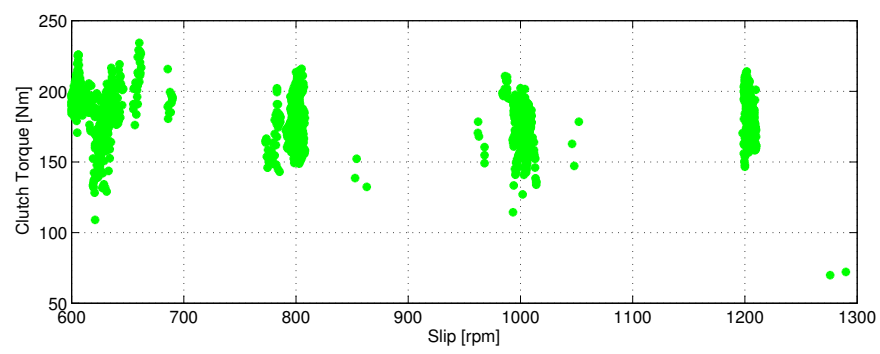

Fig. 4. The data points from Fig. 3 when the engine speed is constant. No correlation is seen between clutch torque and slip speed.

\section{Temperature EFFECTS AND MOdels}

The shape of the torque transmissibility curve is mainly due to the cushion spring characteristics. [8] mentions that this curve can be described by a third order polynomial,

$$
M_{\mathrm{ref}}\left(x_{\mathrm{ref}}\right)= \begin{cases}a\left(x_{\mathrm{ref}}-x_{\mathrm{ref}, \text { ISP }}\right)^{3}+ & \\ +b\left(x_{\mathrm{ref}}-x_{\mathrm{ref}, \text { ISP }}\right)^{2} & \text { if } x_{\mathrm{ref}}<x_{\mathrm{ref}, \text { ISP }} \\ 0 & \text { if } x_{\mathrm{ref}} \geq x_{\mathrm{ref}, \text { ISP }}\end{cases}
$$

where $x_{\text {ISP }}$ (Incipient Sliding Point) is the kiss point, where the push plate and clutch disc first meet and torque can start to transfer. There is no first or zeroth order term in the equation since it is desired to have zero torque and derivative at $x_{\text {ref,ISP. }}$ The exact value of $x_{\text {ISP }}$ can be difficult to find since the transmissibility curve is very flat around $x_{\text {ISP. However it }}$ is only important to find a $x_{\text {ISP }}$ that gives a good curve fit,

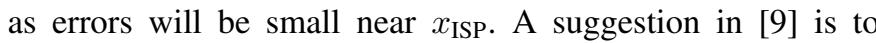
define $x_{\text {ISP }}$ as the point that transmits a certain small torque. That method should give sufficient estimates of $x_{\text {ISP }}$ for the application described here.

Experimental data confirms (5) to be a good approximation for a given temperature, $T_{\text {ref }}$, see Fig. 2. However during slipping of the clutch, heat is dissipated from the friction surface into the cast iron parts of the clutch that naturally will expand. When the actuator motor is fully retracted (position=0) the expansion can be measured through the position sensor on

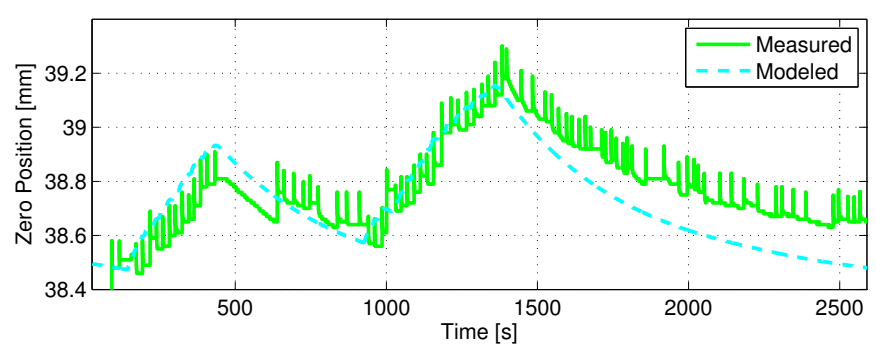

Fig. 5. The clutch has been heated through slipping and then left to cool down. The zero position has been recorded as a measure of the expansion. The temperature model (6)-(9) shows good agreement with the measurement Parameters have been fitted to a separate set of data.

the slave. This slave position is called the zero position, $x_{0}$, see Fig. 1.

A set of experiments has been performed to investigate the dynamics of the clutch expansion. In order to be able to measure the zero position during heating, the clutch has been alternating between closed and slipping with short time intervals. After some time of heating the clutch has been held closed for a prolonged time while the zero position has been constantly monitored as the clutch cools down. The measurement results are found in Fig. 5. The zero position increases in a repeatable way with dissipated energy in the clutch and decays in an exponential manner with time. This supports the hypothesis that temperature is causing the change in zero position.

\section{A. Material Expansion Analysis}

In order to see that the change in zero position is due to material expansion, the temperature increase is calculated in two ways. During the first heating period of Fig. 5, 1.5 MJ of energy is dissipated. The pressure plate and flywheel have a combined mass of $81 \mathrm{~kg}$ and are made of cast iron that has a specific heat of $0.5 \mathrm{~kJ} /(\mathrm{kg} \mathrm{K})$, [14]. If cooling of the clutch is neglected a slightly high estimation of the temperature increase is obtained.

$$
\frac{1.5 \cdot 10^{6}}{500 \cdot 81}=37 \mathrm{~K}
$$

The measured expansion of the clutch during the same period is $0.3 \mathrm{~mm}$ at the piston. With a linkage ratio of 8.8 , a $100 \mathrm{~mm}$ thick clutch and an expansion coefficient for cast iron of 11 . $10^{-6} \mathrm{~K}^{-1}$, [14], this corresponds to a temperature increase of,

$$
\frac{0.3}{8.8 \cdot 100 \cdot 11 \cdot 10^{-6}}=30 \mathrm{~K}
$$

The two temperature estimates are practically the same. Therefore the measured expansion can be said to depend on temperature.

\section{B. Expansion Model}

In order to model the expansion a temperature model is required. In [12] a linear model with one thermal mass in the pressure plate and one in the flywheel is proposed. All parts of the clutch are cooled towards the air in the clutch housing. Here the model is extended to capture the changes in 


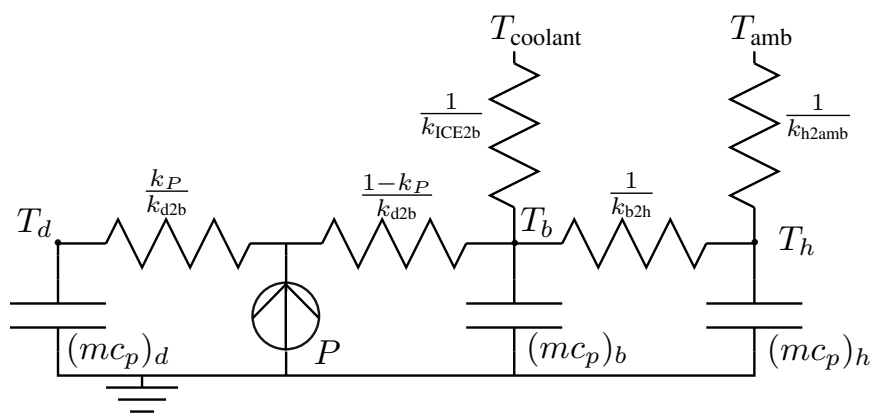

Fig. 6. An electrical analogy of the temperature model (12)-(14). If $\left(m c_{p}\right)_{d}=0$ the model can also be described by (6)-(7).

the housing temperature, $T_{h}$. In contrast to [12] the clutch studied here is mounted in a vehicle, therefore the clutch is, in addition, thermally connected to both the ICE and transmission. However only a few percent of the dissipated power, $P$, goes into the transmission, [13]. Therefore the heat flow to the transmission has been neglected. Moreover it has been found that the thermal masses of the flywheel and pressure plate can be taken as one mass, $\left(m c_{p}\right)_{b}$, for the clutch body, without loosing accuracy with respect to the expansion. For an electrical analogy of the model see Fig. 6 . The corresponding equations are:

$$
\begin{aligned}
& \left(m c_{p}\right)_{b} \dot{T}_{b}=k_{\text {ICE2b }}\left(T_{\text {coolant }}-T_{b}\right)+k_{\mathrm{b} 2 \mathrm{~h}}\left(T_{h}-T_{b}\right)+P \\
& \left(m c_{p}\right)_{h} \dot{T}_{h}=k_{\mathrm{b} 2 \mathrm{~h}}\left(T_{b}-T_{h}\right)+k_{\mathrm{h} 2 \mathrm{amb}}\left(T_{\mathrm{amb}}-T_{h}\right)
\end{aligned}
$$

where,

$$
P=M_{c} \Delta \omega
$$

In order to connect the temperature model with the change in zero position, the levers and the expansion of the clutch body as function of temperature are assumed linear.

$$
x_{0}=k_{\mathrm{exp}, 1}\left(T_{b}-T_{\text {ref }}\right)+x_{0, \text { ref }}
$$

The eight parameters in the equations have been fitted against measured data and the validation results, on a different set of data, can be seen in Fig. 5. Using these results the piston position can be corrected for the expansion effect to correspond to the actual compression of the flat spring, see Fig. 1.

$$
\begin{aligned}
\Delta x_{0} & =x_{0}-x_{0, r e f} \\
x_{\text {cor }} & =x-\Delta x_{0}
\end{aligned}
$$

Nevertheless the expansion model does not explain the torque drift, see Fig. 7. The time scale in Fig. 2 is significantly smaller than that of Fig. 5. The torque drift is also much larger than what is explained by the expansion. This has led to the hypothesis that the clutch disc is also expanding with heat. In particular the clutch disc has a much smaller (thermal) mass, which could correspond to the faster time constant phenomenon seen in the data.

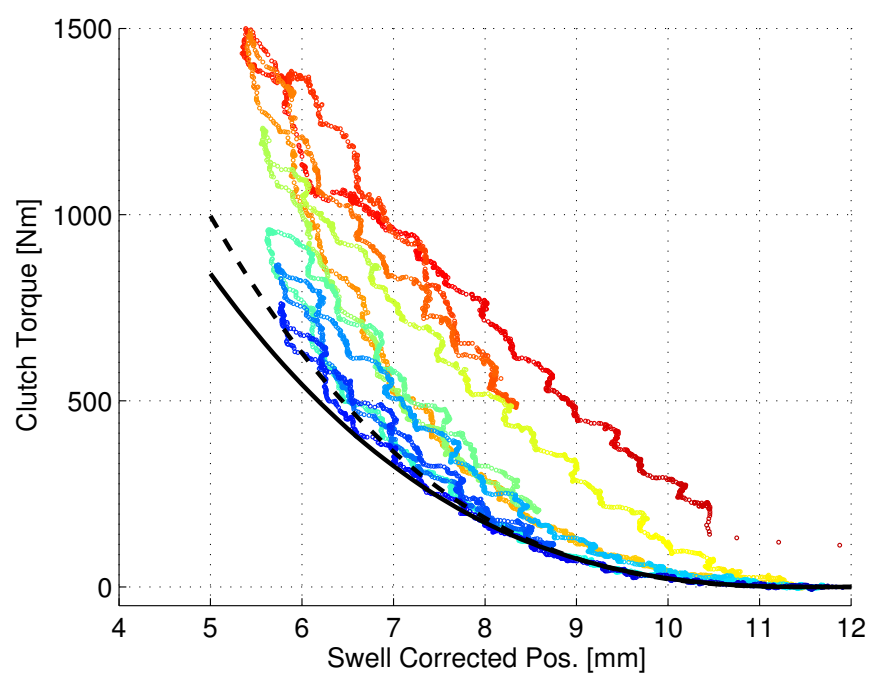

Fig. 7. The data from Fig. 2 has been corrected for swelling of the clutch body using (11). The correction is not sufficient to explain the torque drift.

\section{Including Fast Dynamics}

The clutch disc temperature, $T_{d}$, has been modeled in a similar fashion, see Fig. 6 for an electrical analogy and below for equations.

$$
\begin{aligned}
\left(m c_{p}\right)_{b} \dot{T}_{b} & =k_{\text {ICE2b }}\left(T_{\text {coolant }}-T_{b}\right)+k_{\mathrm{b} 2 \mathrm{~h}}\left(T_{h}-T_{b}\right)+ \\
& +k_{\mathrm{d} 2 \mathrm{~b}}\left(T_{d}-T_{b}\right)+k_{P} P \\
\left(m c_{p}\right)_{h} \dot{T}_{h} & =k_{\mathrm{b} 2 \mathrm{~h}}\left(T_{b}-T_{h}\right)+k_{\mathrm{h} 2 \mathrm{amb}}\left(T_{\mathrm{amb}}-T_{h}\right) \\
\left(m c_{p}\right)_{d} \dot{T}_{d} & =k_{\mathrm{d} 2 \mathrm{~b}}\left(T_{b}-T_{d}\right)+\left(1-k_{P}\right) P
\end{aligned}
$$

A new situation with this extended model is that the dissipated energy has to be split between the clutch disc and the body. In the model it is split by the parameter $k_{P}$, which is 1 if all energy goes to the body and 0 if all goes to the disc. However the model is not sensitive to $k_{P}$ due to two facts. Firstly the vast difference in time constants, a factor of $\sim 50$, between the disc and the body decouples (12) from (14), i.e. $k_{\mathrm{d} 2 \mathrm{~b}}\left(T_{d}-T_{b}\right)+$ $k_{P} P \approx\left(1-k_{P}\right) P+k_{P} P=P$, leaving the dynamics of $T_{b}$ almost unchanged. Secondly the parameters are fitted to data, i.e. if $k_{P}$ is 0 instead of 0.5 the value of $\left(m c_{p}\right)_{d}$ and $k_{\mathrm{d} 2 \mathrm{~b}}$ will double, leaving the dynamics of $T_{d}$ almost unchanged. In the data the expansion has been seen to lag the dissipated power. The lag is maximized by $k_{P}=0$ this is thus the choice of $k_{P}$

The expansion of the clutch disc is modeled by extending (9) with an extra term

$$
\begin{aligned}
\Delta x_{0} & =k_{\exp }\left(T_{b}-T_{\text {ref }}\right)+k_{\text {exp }, 2}\left(T_{d}-T_{\text {ref }}\right)= \\
& =k_{\exp }\left(T_{b}-T_{\text {ref }}\right)+k_{\exp , 2}\left(\left(T_{d}-T_{b}\right)+\left(T_{b}-T_{\text {ref }}\right)\right)= \\
& =\underbrace{\left.k_{\exp }+k_{\text {exp }, 2}\right)}_{k_{\text {exp }, 1}}\left(T_{b}-T_{\text {ref }}\right)+k_{\exp , 2}\left(T_{d}-T_{b}\right)
\end{aligned}
$$

where the second term was practically zero during the zeroposition measurements due to the fast dynamics of $T_{d}$. Therefore the new parameters can be estimated separately from the 


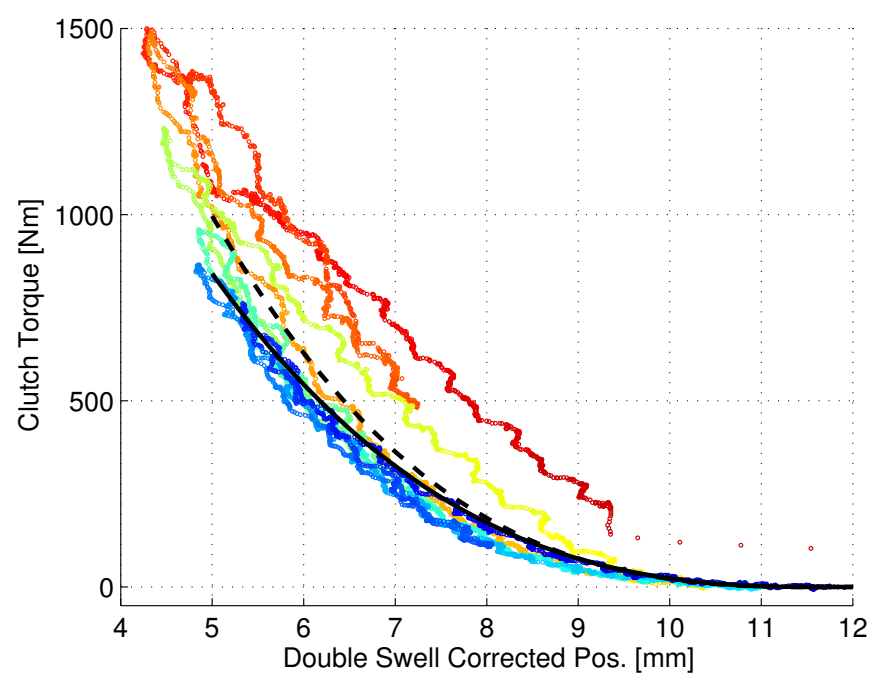

Fig. 8. The data from Fig. 2 has been corrected for swelling of the clutch body using (11) and (15). The correction explains the torque drift until the temperatures get too high (somewhere when state $T_{d}$ becomes greater than $\left.200{ }^{\circ} \mathrm{C}\right)$.

old parameters. After estimation of the new parameters the position can be corrected with (12)-(15) and Fig. 8 is obtained.

The model works well until the temperatures become too high (state $T_{d}$ reaches more than $200{ }^{\circ} \mathrm{C}$ ). It has been found empirically that saturating the term $\left(T_{d}-T_{b}\right)$ at $110{ }^{\circ} \mathrm{C}$ improves the model at higher temperatures. One should note that values of the temperature states have not been validated against any measurement but they have reasonable magnitudes.

The transmitted torque can now be calculated as,

$$
M_{c}=M_{\text {ref }}\left(x_{\text {cor }}\right)
$$

Note that $x_{\text {cor }}$ increases with temperature which in turn makes $M_{c}$ increase with temperature. This is in contrast with the data in [10] where the torque is decreasing with temperature. Although in the experiments performed here the clutch mostly has a medium to high temperature and could comply with the data in [11].

In summary, the model is a three state model with one static non-linearity. All calculations are simple and therefore the model is suitable for running in real time, for example in a clutch-control application.

\section{Model VALidation}

The model has been validated on several data sets separate from those used for parameter identification. One of these validations is shown in Fig. 10. The model can be said to agree well with data. If the dissipated power is calculated from the measured torque the model agrees very well, but it still simulates a slightly low clutch torque. If instead the dissipated power is calculated from the simulated torque the torque error makes the simulated power dissipation too low and therefore the temperature states get lower. In turn this will lead to even lower simulated torque in the next torque pulse. This is the explanation for the decay in the simulated torque.

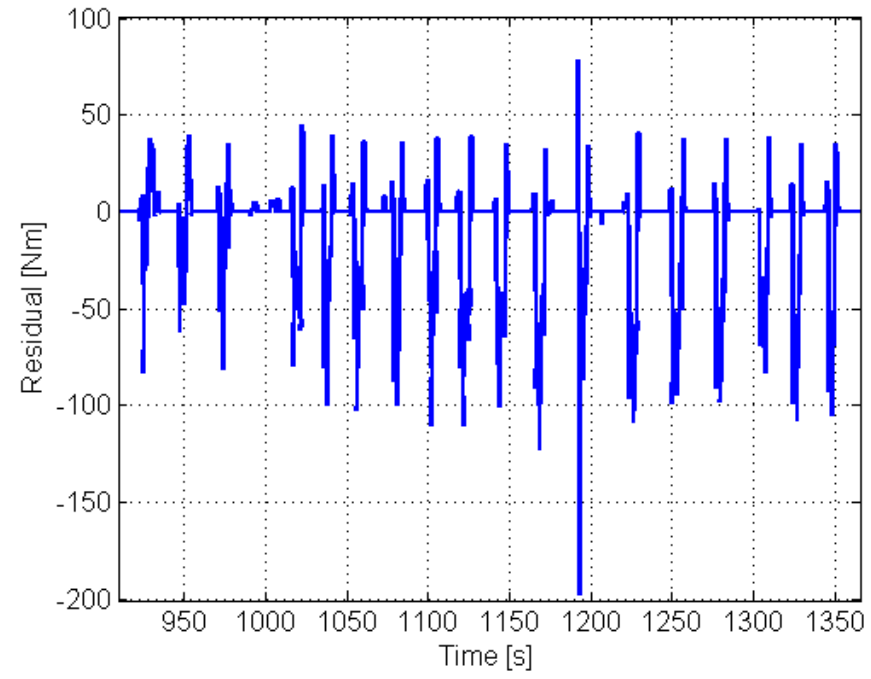

Fig. 9. The residuals between the model and data presented in Fig. 10. The residuals are less than $200 \mathrm{Nm}$ and most of the time less than $100 \mathrm{Nm}$. Or in other words, most of the time less than $3 \%$ of maximum ICE torque.

In spite of this it still models the torque better than the more common static model. On one hand, calculating the power from the measured torque gives a better estimate of the current transmissibility curve, which could be used for feed forward in control applications. On the other hand the measured torque is not always available, e.g. some special driving scenarios, when evaluating new controllers through simulation, or in the prediction made by a model-based controller.

At the end of the torque pulses, the modeled torque drops faster than the measured torque, when the clutch is fully opened in $0.3 \mathrm{~s}$. This is however believed to be due to delays in the computation of the engine torque, sensors and other data processing. Therefore the residuals have been filtered using a moving median of five samples $(10 \mathrm{~Hz})$. The result is presented in Fig. 9. The residuals are less than $200 \mathrm{Nm}$ and most of the time less than $100 \mathrm{Nm}$. Considering that $100 \mathrm{Nm}$ is less than $3 \%$ of maximum engine torque, the model works well. In fact the engine estimated torque can have errors of similar magnitude.

A validation of a launch is shown in Fig. 11. The static and dynamic models are compared to measurement data. The dynamic model has a small error that is always less than $104 \mathrm{Nm}$, and has better performance than the static model.

In other data sets it has been seen that somewhere when $T_{d}$ is greater than $200{ }^{\circ} \mathrm{C}$ the model deteriorates. This however is of small concern since these temperatures are only reached during extreme driving. The accuracy of the model has also been seen to be less good when the truck is cold.

The clutch temperatures could not be quantitatively validated as no temperature measurements were available. Therefore only a qualitative validation is performed, showing that the magnitudes of the temperature states are reasonable for an HDT clutch, see Fig. 10 and 11. 

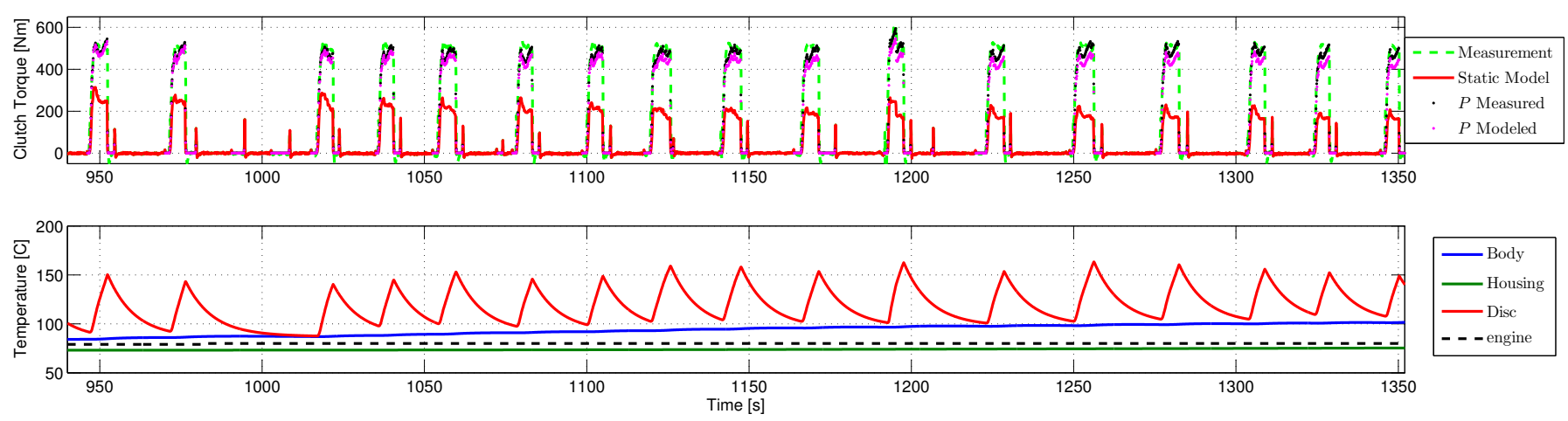

Fig. 10. The dynamic torque model shows good agreement with measurements in a validation data set. There is a slight decay in torque when $P$ is modeled instead of measured. However both implementations are significant improvements compared the static model, $M_{c}=M_{\text {ref }}(x)$. The open-loop simulation started at 0 seconds. In the lower plot $P$ is measured.
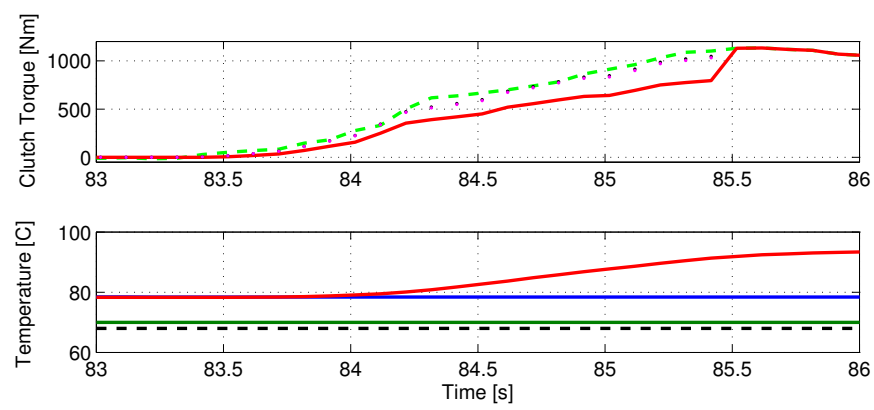

Fig. 11. The dynamic torque model shows good agreement with measurement data of a start. The open-loop simulation started at 0 seconds. Line colors and styles are the same as in Fig. 10. The clutch is locked up when there are no black or magenta dots. In the lower plot $P$ is measured.

\section{CONCLUSION}

The transmitted torque in an heavy-duty truck dry clutch at slipping conditions has been studied. Experiments showed no direct slip-speed dependency at the levels of slip investigated here, instead dynamic effects were clearly visible. In particular the measurements showed that the transmitted torque can vary with up to $900 \mathrm{Nm}$ for a given position. To capture the dynamic behavior, a model with three temperature states was built. The model was developed and validated on a production HDT, were no temperature measurements were available. Therefore only a qualitative validation of the temperatures was performed, showing that they had reasonable magnitudes.

The temperature dynamics have two vastly different time constants, a factor of $\sim 50$ apart, and the slow part could be physically explained to affect the transmitted torque through expansion of clutch parts. Due to the vast difference in the two time constants the model shows little sensitivity to how the dissipated heat enters the model.

In validations, on driving sequences with intense use of the clutch, the open-loop simulation error was kept under $200 \mathrm{Nm}$ and most of the time under $100 \mathrm{Nm}$, which is less than $3 \%$ of maximum engine torque and comparable to the accuracy of the engine torque estimate. The dynamic model was shown to give a significant improvment compared to a static model.
Finally, the model is simple and suitable for running in real time applications, such as a clutch controller or a torque observer.

\section{ACKNOWLEDGMENT}

Vinnova industrial excellence center LINK-SIC and Scania CV have supported this work. Special thanks to Karl Redbrandt at Scania for conducting the experiments.

\section{REFERENCES}

[1] B. Mashadi and D. Crolla, Vehicle Powertrain Systems, 1st ed. John Wiley \& Sons Ltd, 2012.

[2] F. Vasca, L. Iannelli, A. Senatore, and G. Reale, "Torque transmissibility assessment for automotive dry-clutch engagement," IEEE/ASME transactions on Mechatronics, vol. 16, no. 3, pp. 564-573, June 2011.

[3] P. Dolcini, C. C. de Wit, and H. Béchart, "Lurch avoidance strategy and its implementation in AMT vehicles," Mechatronics, vol. 18, no. 1, pp. 289-300, May 2008.

[4] F. Garofalo, L. Glielmo, L. Iannelli, and F. Vasca, "Optimal tracking for automotive dry clutch engagement," in 2002 IFAC, 15th Triennial World Congress, 2002.

[5] P. Dolcini, C. C. de Wit, and H. Béchart, "Improved optimal control of dry clutch engagement," Proceedings of the 16th IFAC World Congress, vol. 16, no. 1, 2005

[6] M. Bataus, A. Maciac, M. Oprean, and N. Vasiliu, "Automotive clutch models for real time simulation," Proceedings of the Romanian Academy, Series A, vol. 12, no. 2, pp. 109-116, 2011.

[7] L. Glielmo and F. Vasca, "2000-01-0837: Optimal control of dry clutch engagement," in SAE 2000 World Congress, March 2000.

[8] P. J. Dolcini, C. C. de Wit, and H. Béchart, Dry Clutch Control for Automotive Applications, ser. Advances in Industrial Control. SpringerVerlag London, 2010.

[9] G. Mattiazzo, S. Mauro, M. Velardocchia, F. Amisano, G. Serra, and G. Ercole, "2002-01-0934: Measurement of torque transmissibility in diaphragm spring clutch," in SAE 2002 World Congress, March 2002.

[10] M. Velardocchia, G. Ercole, G. Mattiazzo, S. Mauro, and F. Amisano, "1999-01-0737: Diaphragm spring clutch dynamic characteristic test bench," in SAE international congress 1999, March 1999.

[11] G. Ercole, G. Mattiazzo, S. Mauro, M. Velardocchia, F. Amisano, and G. Serra, "2000-01-1151: Experimental methodologies to determine diaphragm spring clutch characteristics," in SAE 2000 World Congress, March 2000.

[12] M. Velardocchia, F. Amisano, and R. Flora, "2000-01-0834: A linear thermal model for an automotive clutch," in SAE 2000 World Congress, March 2000.

[13] A. Wikdahl and §̊. Ågren, "Temperature distribution in a clutch," Master's thesis, Linköping University, 1990.

[14] C. Nording and J. sterman, Physics Handbook. Studentlitteratur, 1999. 